\title{
Domino: Twin interfaces to unveil the influence of personality traits on usability
}

\author{
Hubert Smeitink \\ Eindhoven University of Technology \\ Department of Industrial Design \\ Eindhoven, the Netherlands \\ h.smeitink@gmail.com
}

\author{
Joep Frens \\ Eindhoven University of Technology \\ Department of Industrial Design \\ Eindhoven, the Netherlands \\ j.w.frens@tue.nl
}

\section{Abstract}

This pictorial describes the design and design process of the Domino interfaces: a pair of light interfaces consisting of a tangible user interface and its touchscreen equivalent. This pair of user interfaces is designed to be used in an exploratory comparative study concerning the influence of personality traits on perceived usability. We present the design process of this pair of user interfaces, focussing on the unique challenges of designing a functionally equivalent set of physical and graphical user interfaces. The exploratory comparative study, which utilizes these Domino interfaces, is presented next. We continue with the results from the study after which we conclude with a concise discussion and outlook on future work.

\section{Authors Keywords}

Interface Design; Tactile Interaction; Graphical User Interface; Physical User Interface; Personality Traits

Permission to make digital or hard copies of part or all of this work for personal or classroom use is granted without fee provided that copies are not made or distributed for profit or commercial advantage and that copies bear this notice an the full citation on the first page. Copyrights for third-party components of th work must be honored. For all other uses, contact the Owner/Author.

TEI '22, February 13-16, 2022, Daejeon, Republic of Korea (C) 2022 Copyright is held by the owner/author(s). (1)

\section{CSS Concepts}

-Human-centered computing Interaction

design $\sim$ Interaction design process and methods User interface design

-Human-centered computing Interaction

design Empirical studies in interaction design

\section{Introduction}

Touchscreen interfaces and voice commands are becoming an increasingly popular alternative to physical user interfaces for both users and manufacturers. Thermostats, light switches, and even the washing machine lose their physical buttons in favour of voice commands, touchscreen interactions, or automated control (e.g. [24][25][26]). This shift to virtual interaction elements brings with it advantages in flexibility and upgradability, but transitioning away from physically rich user interfaces also has its downsides. Research has shown that physical user interfaces can outperform their touchscreen counterparts measured both objectively in productivity, as well as subjectively in the form of perceived usability [11][23][18]. The objective side, in the form of productivity metrics, has received significant attention from $\mathrm{HCI}$ researchers [9][22][15]. While the subjective side of usability has its place in literature [2][20] the relation between preference and interaction style has received little attention. This leads us to the question: Are there differences in preference for physical and graphical user interfaces, and can these differences be explained by specific personality traits?

Two personality traits stood out in particular due to their likely influence on perceived usability: the effect of what we have called 'tactile appreciation' and the influence of holistic versus analytical perception [19]

To assess the effects of both these traits, we designed a set of two user interfaces specifically to be used in a comparative test: one physical user interface and a functionally equivalent graphical alternative.

In this pictorial, we will focus specifically on the design process of the physical user interface. We present the functionally equivalent graphical user interface only as a result. Designing this physical user interface brought along unique challenges, as features and options for implementation needed to be carefully selected to prevent an inherent favour for either of the two user interfaces: physical user interfaces excel in different things than graphical user interfaces do and we needed to ensure that there was no inherent bias towards the one or the other interface. This was complicated by the fact that functions that are easy to implement in a graphical user interface are often hard to realize in a physical alternative, requiring innovative mechanical solutions like those presented in this pictorial. To round off this design journey, we end this pictorial with an exploratory comparative study, utilizing both user interfaces to evaluate the effects of the two personality traits, as described next. 


\section{Tactile appreciation}

In the past, before the advent of electronic appliances, purely mechanical products naturally instil the user with an understanding of its functioning [8]. Wensveen et al. [21] describe this information flow in his 'froggerframework', where it is split into feedforward and feedback information types. The feedforward information is presented to the user before interacting (i.e. the affordance), while the term feedback captures the information resulting from the interaction.

With the introduction of digital (and in particular touch) interfaces came a reduction in this natural feedforward and feedback [1][5]. A touch control may clearly af-

ford pressing, but its result can be ambiguous without clear labelling. Furthermore, the feedback supplied by the touch control is naturally limited, and can provide a potentially false sense of action; Pressing harder won't make an appliance turn on faster, and if unplugged won't even result in turning it on at all.

The reduction in physical feedback is taken even further with the introduction of touchscreen interfaces; The virtual buttons on a touchscreen lack not only natural feedforward information but also natural tactile feedback. Adding artificial haptic feedback can remedy this to an extent, but cannot take away the reduction in dimensionality. The fact that a touchscreen only offers a flat surface to interact with limits the usage of our naturally developed skills to interact with objects in three dimensions, using our hands. It limits the user to at most the several fingertips touching a flat and solid surface.

Both the lack of three-dimensional physical manipulation and reduced tactile feedback can potentially negatively impact the perceived usability of the user interface. The extent to which this perceived usability is altered may very well differ from person to person, depending on their appreciation of these tactile interaction properties. We will refer to this as tactile appreciation.

\section{Holistic versus analytical perception}

The way in which we perceive the world, on a scale from holistic to analytic, varies between individuals. Research has shown that the average way in which people perceive the world differs between cultures [16]. North Americans tend to focus both mentally and physically on the same object, while being relatively inattentive to the context surrounding this object [19]. This type of perception is classified as the analytical type.

The opposite was observed in Asian culture, where the context surrounding an object plays a bigger role due to their (on average) more holistic, field dependent, perception. These differences in perception are not necessarily limited to an intercultural level, but might also exist between classes of society:

"... Eastern Europeans showed more context-dependent attentional patterns than did Western Europeans and that Southern Italians and working-class Italians showed more context-dependent reasoning styles than did Northern Italians and middle-class Italians"

In accordance with these observations, recognition tasks showed that Eastern participants performed worse than Western participants when the background in object recognition tasks was altered (as this disturbed the holistic image they had formed).

Extending this train of thought the possibility arises that even within one culture, holistic or analytical perception could impact the perceived usability of user interfaces that are more holistic or more analytic in nature.

As GUI's need to display all their information on a plane that can be viewed in one glance, it could be argued that they tend to offer a more holistic overview than TUI alternatives.

TUI alternatives, on the other hand, tend to exist of a multitude of physically separated and dimensionally richer control elements, making them potentially better suited for an analytical perception.

\section{An explorative comparative study}

To examine the potential influence of these two personality traits on the perceived usability of graphical user interfaces and their tangible alternatives, we set out to conduct an explorative comparative study. For this study, both a purely physical and a touchscreen interface needed to be developed.

The design process for both these user interfaces started with the formulation of three main requirements:

1. Both interfaces need to offer the same functionality.

2. The visual appeal of both interfaces should be rated on average, as being equal.

3. The usability of both interfaces should, on average, be rated the same.

These three requirements ensure a fair comparison in which neither user interface is inherently favoured as a result of design decisions; If one of the user interfaces were to constantly score higher on visual appeal or usability, then this fact alone could potentially explain higher perceived usability.

Having formulated the requirements, the next step in the design process consisted of deciding on the type of control that would be offered through the user inter-

faces. It was deemed best to choose an interaction goal with which we are all familiar, in order not to add unneeded complexity to the user interface comparison. This resulted in the decision to have both user interfaces control the lights within a living room. Or, more specifically, the lights in the IoT Sandbox shown next. 


\section{The loT Sandbox}

This study is part of a series of studies that make use of the same experimentation platform: a scale model of a smart home named the IoT Sandbox [7], see figure 1. The IoT Sandbox provides a concise, movable and affordable platform for experimentation on interaction styles and offers a series of connected systems that are accessible through experimental user interfaces. It alleviates the need to build custom demonstrators while still offering high quality system feedback thus focusing the effort of setting up the study towards making high quality user interfaces. While the IoT Sandbox platform has been used and published on before, the interface described in this pictorial is novel.

\section{Targetable light strip}

The IoT Sandbox is equipped with several connected systems: it has streaming audio in multiple zones, streaming video, automated blinds and a series of light fixtures throughout the house as well as a targetable light strip in the living room. Only the targetable light strip is used in this study. This strip consists of a series of individually controllable RGB LEDs, allowing for fine positional control of the light along the U-shaped strip. The user interfaces designed for this study will be placed in front of the IoT Sandbox as shown in figure 1.
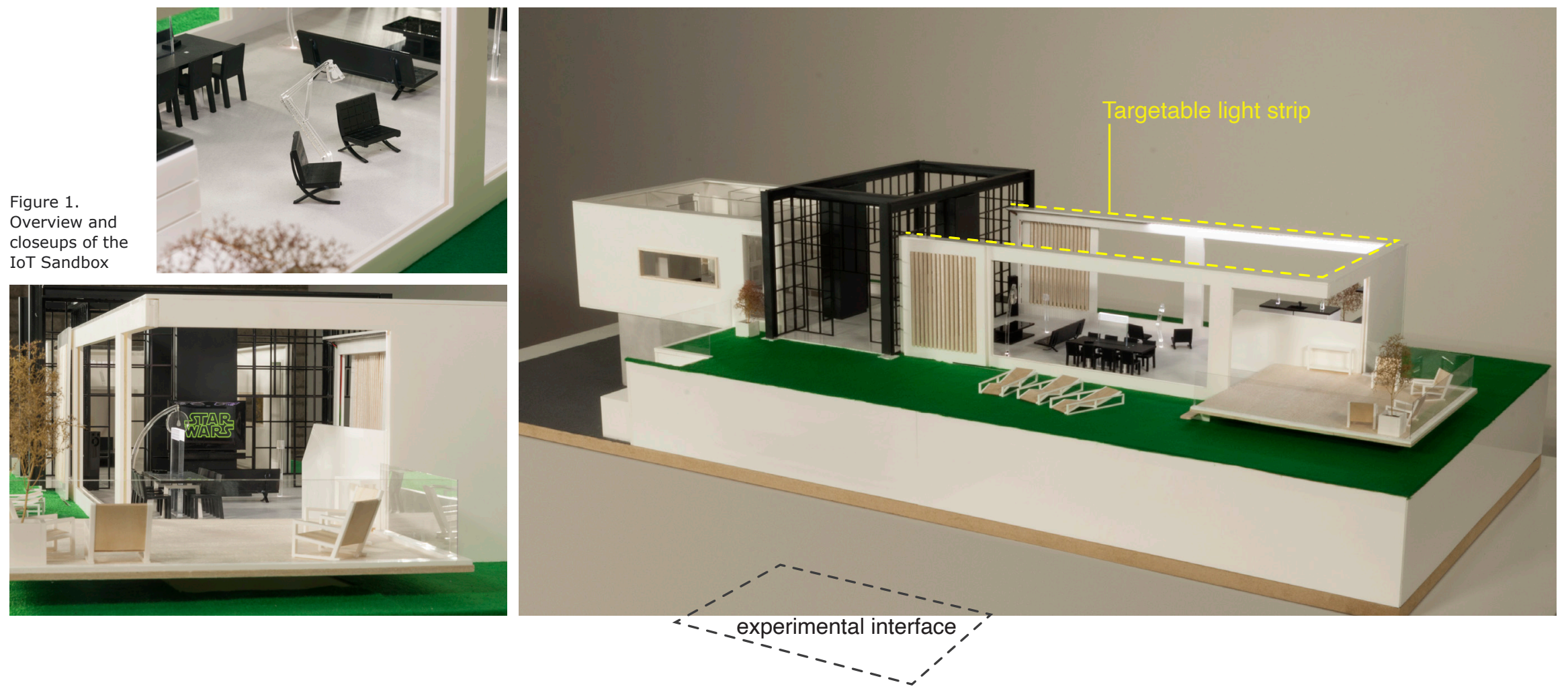


\section{Creating a lever}

Apart from adhering to the shared requirements for both user interfaces, the physical user interface needed to capitalize on its potential for rich, tactile, interaction to differentiate itself from the touchscreen-based alternative. To do so, we build on a concept from Lesley Lock and Simon Bavinck, that uses an array of rotating blocks to control light rather than simple linear switches or sliders. Instead, inspired by power controls such as those found in airplanes, a lever mechanism was devised. Pushing this lever one way increases the light while pulling it the other way reduces it.

A considerable amount of attention was given to the feeling of the lever movement. Creating physical and functional prototypes during the first iterations proved essential to test and tune this feeling and the quality of the resulting interaction.

In tandem with the development of the physical user interface, the first iterations of the graphical user interface also came into existence. These early iterations lead to the realization that omitting a central on/off control felt like an artificial limitation to the graphical user interface. After all, adding such a virtual button is a relatively simple task. This, however, could not be said for the physical user interface. The lever positions map directly to the intensity of the lights. Turning the lights on or off without moving the levers would break this mapping. A solution was found in the addition of actuated levers with a custom friction clutch design.
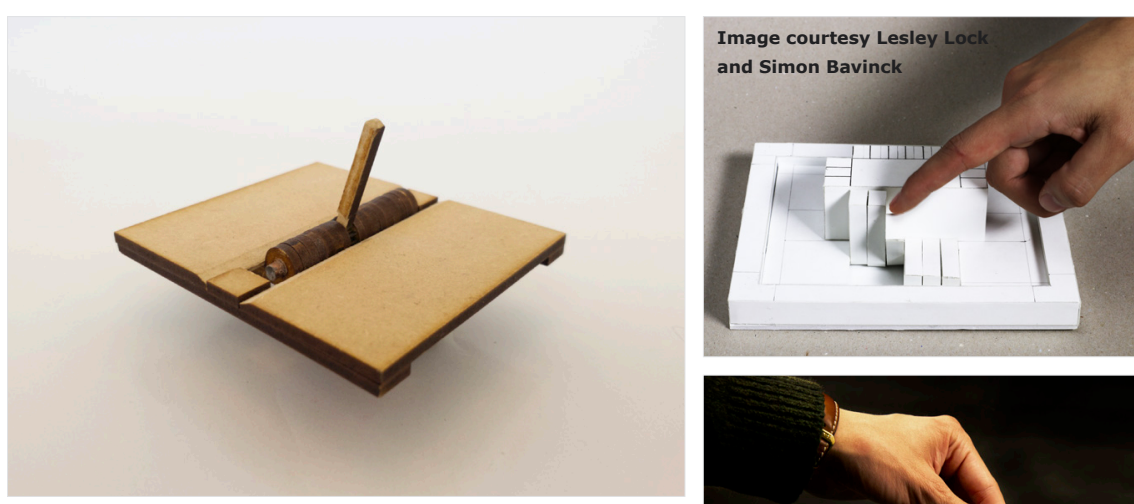

Figure 2. An overview of the original cardboard prototype by Lesley Lock and Simon Bavinck, and succeeding functiona iterations; Transitioning from MDF to plexiglass for tighter plexiglass for tighter tolerances and better
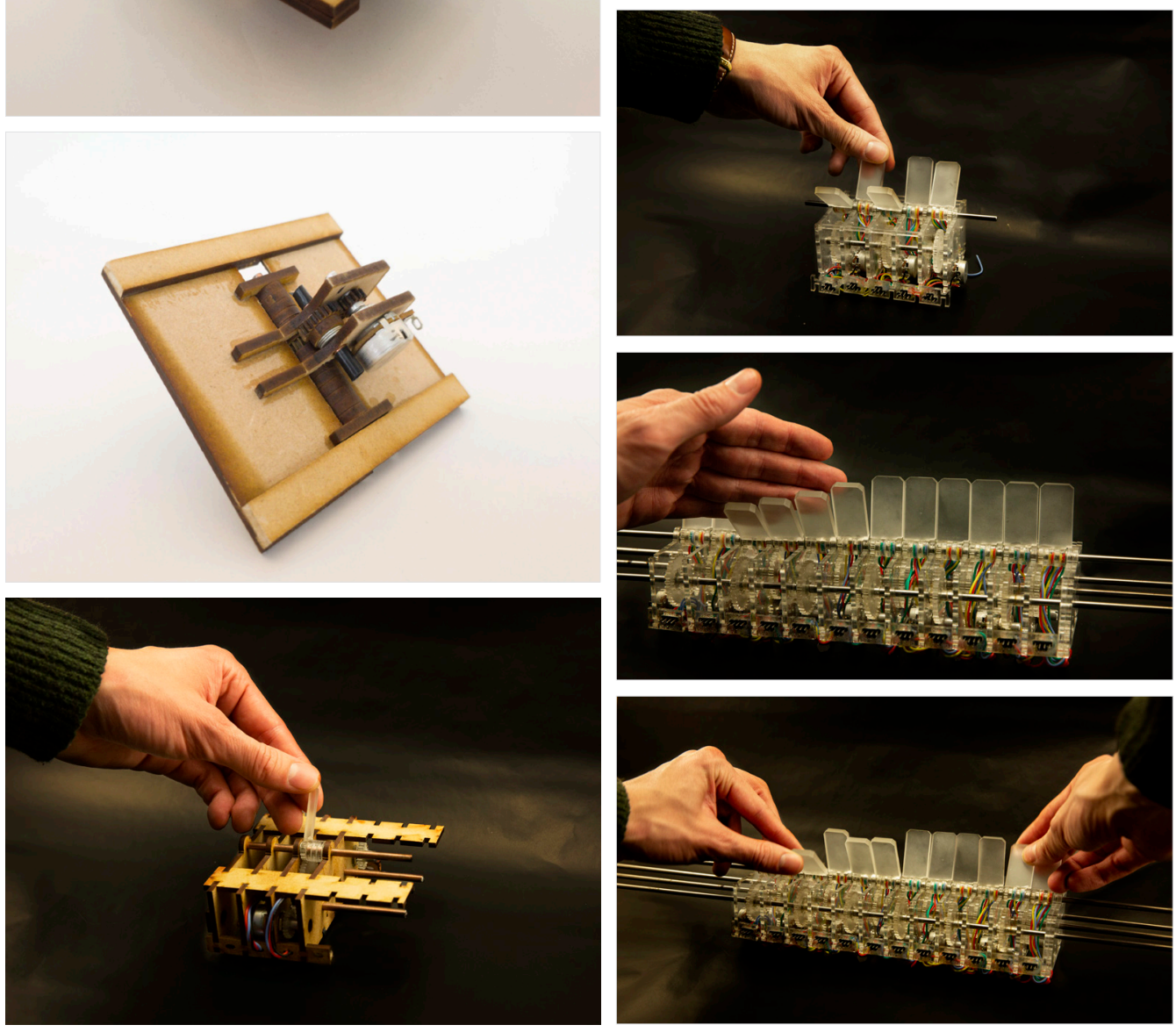


\section{Manual \& Automatic}

Designing a mechanism that allows for both automatic and manual movement of the lever, while retaining the feeling of the lever, proved to be a significant challenge. Merely connecting the motor directly made turning the lever too hard, so a custom friction clutch was introduced, figure $3 \& 4$. This mechanism consists of a hollow axle, on which two gears are placed (figure 5). The bottom gear can spin around the hollow axle and provides a direct coupling between a potentiometer and the lever. The top gear is glued to the shaft and driven by the motor.

A custom made belleville spring induces a dispersing force between the two gears, causing friction between the spinning gear and the friction disc on the flange at the bottom of the hollow axle. The retaining clip on the top of the shaft prevents the top gear from slipping upwards, keeping all parts (figure $6 \& 7$ ) in place.
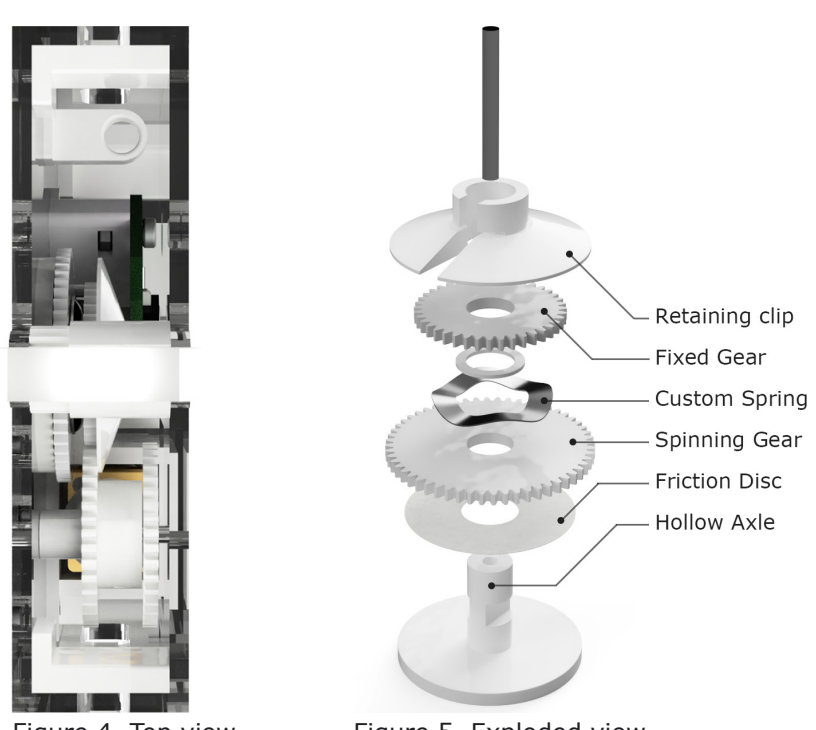

Figure 5. Exploded view

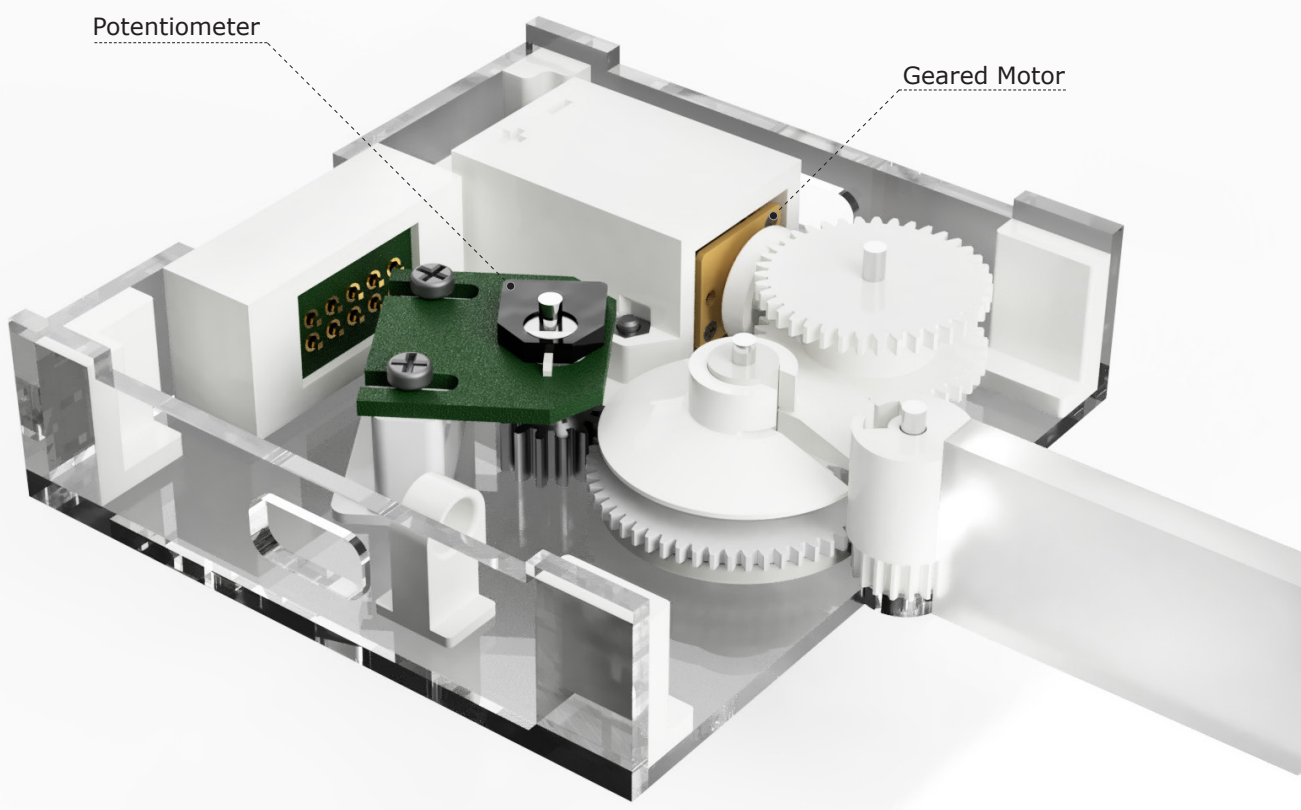

Figure 3. Render of the lever mechanism

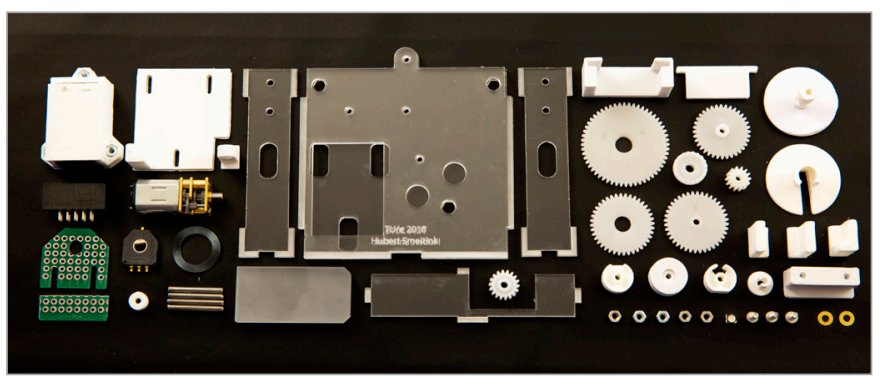

Figure 6. Parts overview

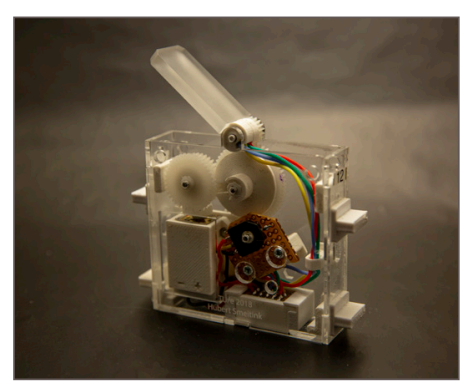

Figure 7. Assembled lever 


\section{The feel of a lever}

The new friction clutch mechanism brought with it the friction disc. Without this disc the direct contact between the nylon gear and the bottom flange causes significant stick-slip. The friction disk serves to eliminate this effect and provides a way to tune the feel of the lever. Adding a thicker disc increases the force required to turn the lever, and different materials cause varying amounts of stick-slip.

Since the tactile experience of the lever is a crucial aspect of the physical user interface, we experimented with a wide range of materials for this disc. In the end, we narrowed the selection down the eight most promising materials shown in figure 8 . In the end, the rough paper disc proved to provide the best tangible experience.

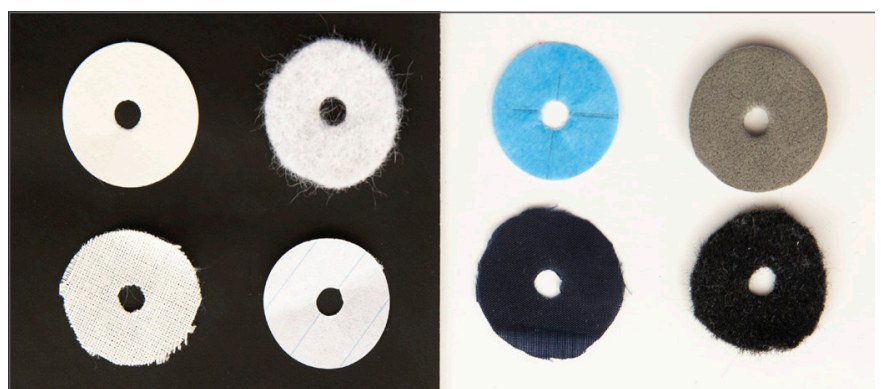

Figure 8. Friction materials tested for the clutching mechanism.

From top left to bottom right: Thick rough paper, thin synthetic felt, painters tape, foam, course fabric, thing regular paper, fine fabric, thick synthetic felt.

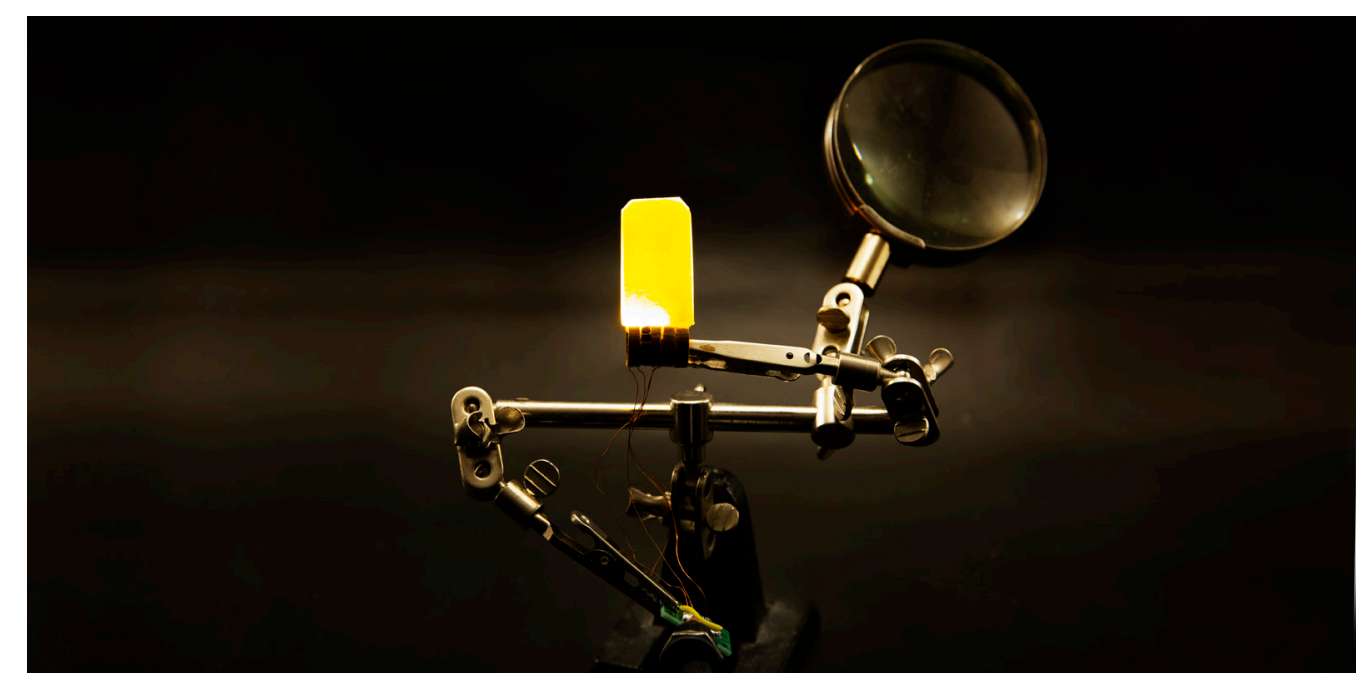

\section{A tangible light interface}

As part of an internship, students Lesley Lock and Simon Bavinck designed alternative rich [8] and modular light interfaces for the existing IoT Sandbox [7]. One of their designs (see image 2) struck us as important as it elegantly showed how rich user interfaces can offer a direct handle on functionality. The design was unusable for their modular tile interface and was therefore shelved. The design was revived for this project and offered valuable inspiration of how to integrate form, interaction and function [8] in the tangible light interface.

Arranging the levers in the same $U$ shape as the light strip they control helps the user to understand the mapping from levers to lights intuitively, but on its own does not remedy the disparity in lever and light placement. Since we cannot practically bring the lever to the light, we instead brought the light to the levers. By adding an RGB led in the base of the sandblasted lever we were able to light it up in the same way as the light it controls, thus providing a representation of the effect of the lights in the room (figure 9).
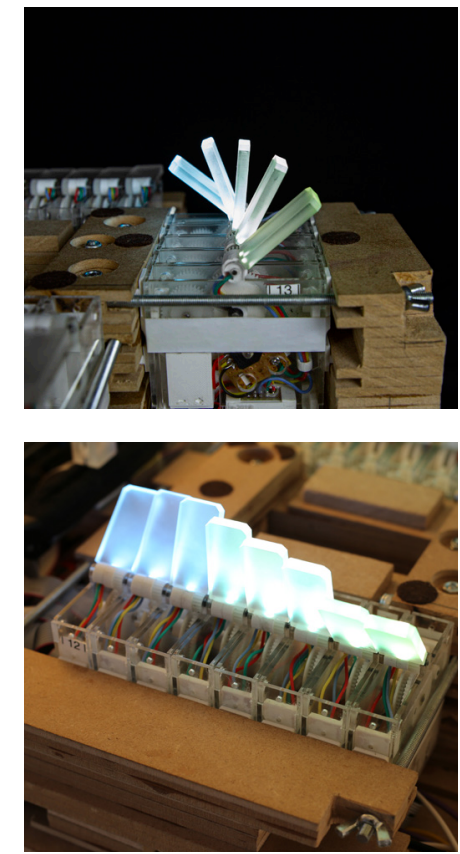

Figure 9. Adding light to the levers 


\section{Domino: its physical form}

The final version of the physical Domino interface is finished by a reflective black outer shell to accentuate the light of the levers. The Domino interface has a total of 21 physical

levers arranged in the same shape as the light they control.

A slider to control the brightness of all levers simultaneously

is located on the left side of the interface, see figure 10 .

\section{Technology}

The black shell of the physical Domino interface rests on an MDF framework. This framework holds the rows of levers, as well as the slider and electronics. The electronics consist of 2 customdesigned printed circuit boards. Each of these boards contains a set of motor drivers, led drivers, and analogue multiplexers to channel the positional feedback from the lever modules. A Teensy 3.6 microcontroller controls both boards.

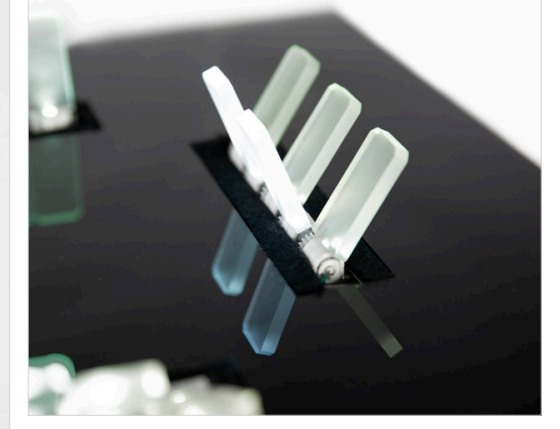

Figure 10.

Overview and closeups of the physical Domino user interface

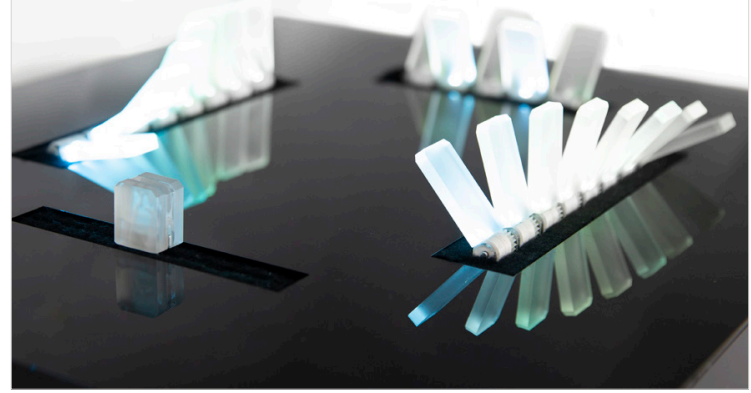




\section{Mapping levers to lights}

The lever in the physical Domino interface can be viewed as handles allowing the user to literally pull light into the room by turning them inwards towards the centre of the room. The physical size of the translucent levers was expressly chosen to allow for a rich tactile interaction using one or even both hands to mould the light. To maximize the expressiveness of the lighting in the model house, the colour temperature of the lights corresponds with the light intensity. The colours range from dim and warm to bright and more blue light, and are equally represented in the colours of the levers themselves, see figure 11 .

\section{Overall brightness control}

Manually turning off the light section on the graphical user interface felt artificially limited, and so overall brightness touch controls were added. The same functionality needed to exist in the physical Domino interface, resulting in the overall brightness slider (figure 12). This slider normally rests in the centre position, and can be moved up to increase the overall brightness, or down to decrease the overall brightness.

During this movement, the relative positions of the levers are kept, and upon release the motorised slider automatically returns to its centre position. The speed at which the levers turn corresponds with the slider's distance from its centre position, allowing for both fine and fast adjustments.
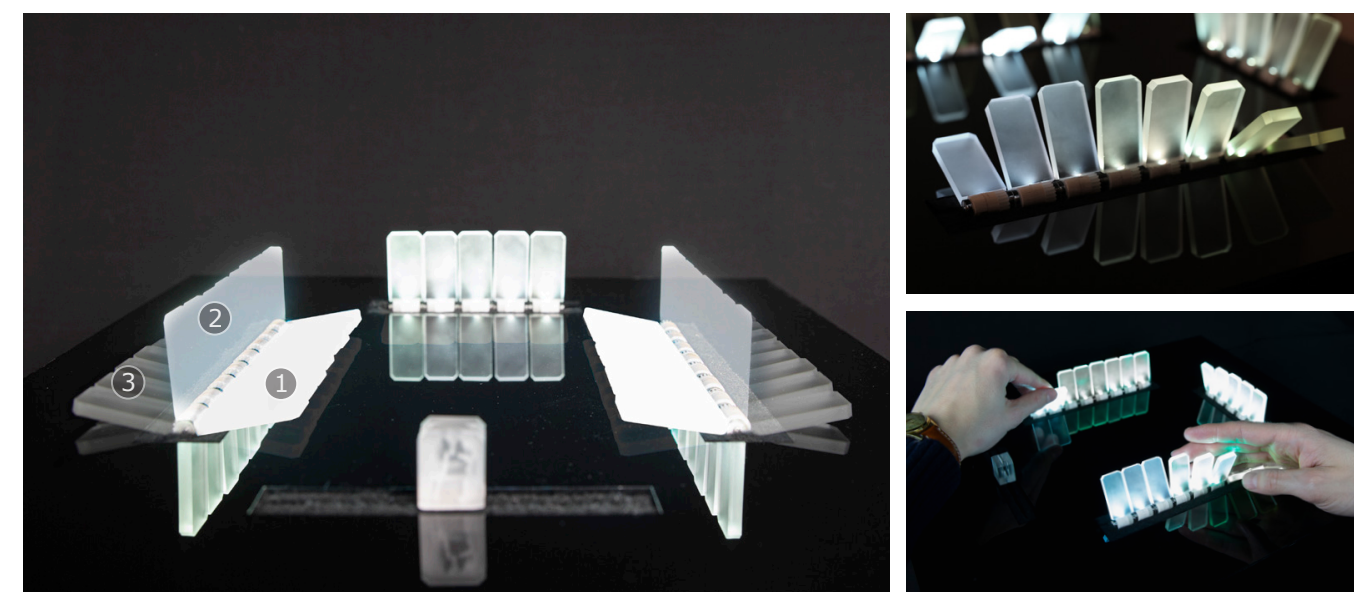

Figure 11. Brightness sections: On (1), Dimmed (2), Off (3)
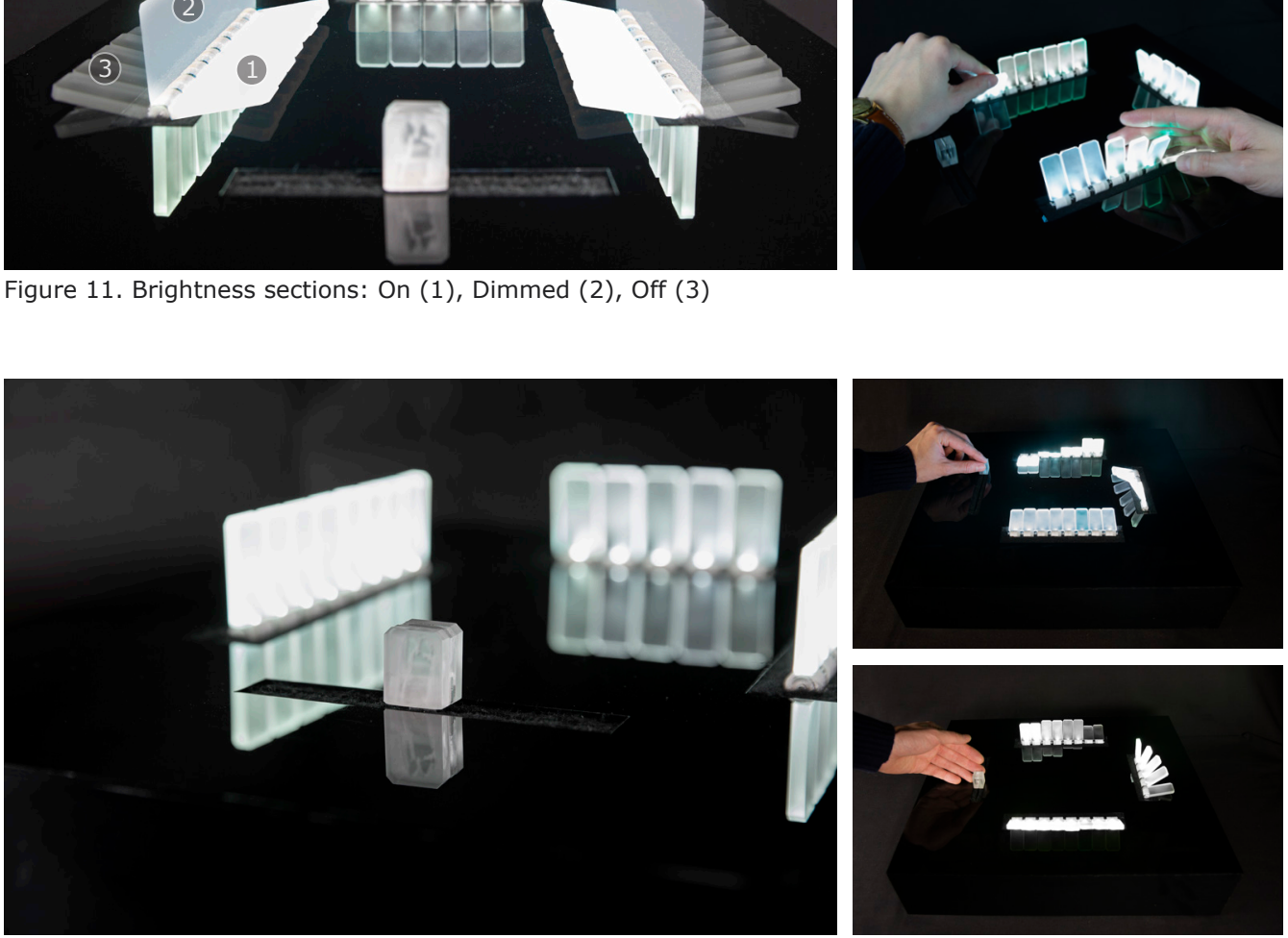

Figure 12. A slider for overall brightness control 


\section{Domino: its graphical form}

The graphical version of the Domino interface is functionally equivalent to its physical counterpart. It offers the user a set of sliders, again positioned in the shape of the lights they control. The amount of light produced by the slider can be seen on the slider itself. The effect on the lights in the room is shown by the light curtain it moves, mimicking a top-down view of the physical version, see figure 13.
Overall brightness controls are implemented using virtual

buttons, offering both relative brightness and fast on/off control, thereby forming a touchscreen friendly alternative to the slider on the physical Domino interface.
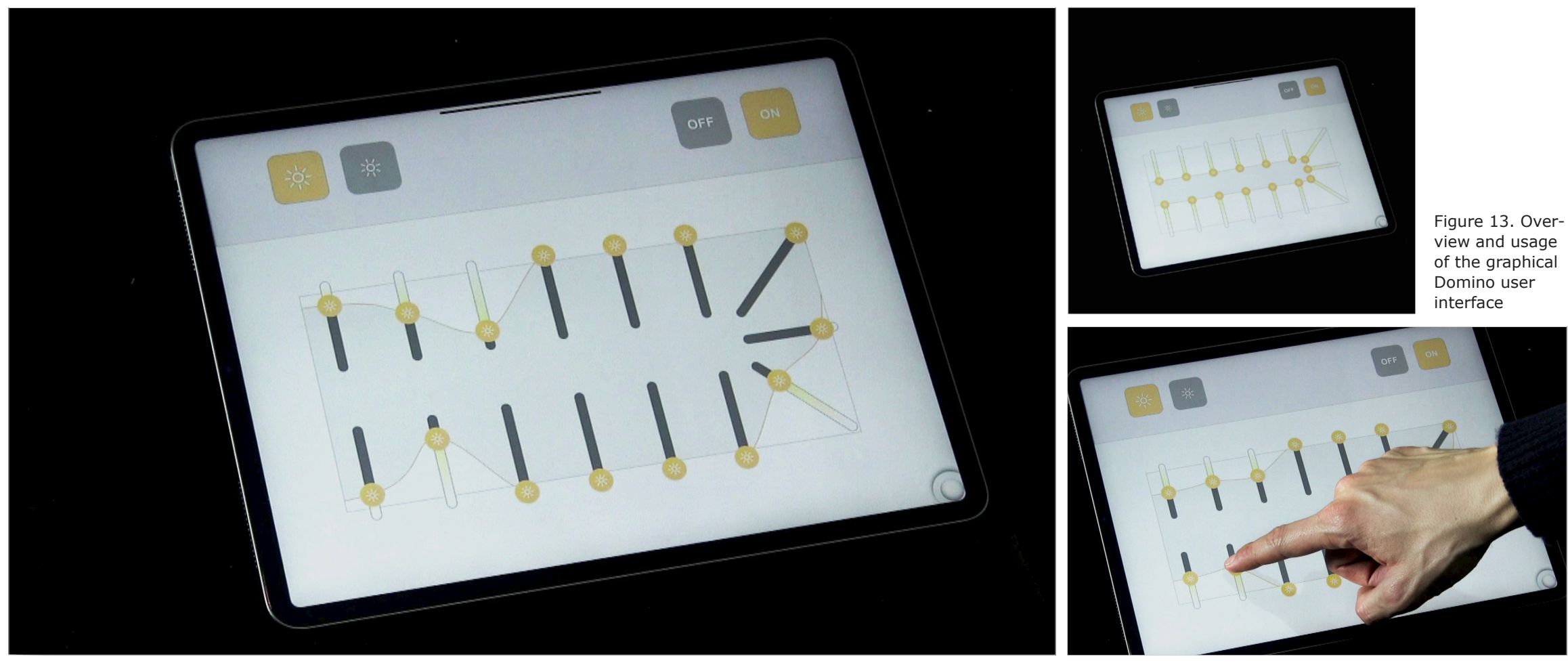


\section{Exploratory comparative test}

We conducted an exploratory comparative study to gain insights into the type of results a full study might reveal, and to potentially further direct the focus of such a study. We recruited a total of 16 participants, aged between 18 and 28 , all from outside our department of Industrial Design to prevent bias. To eliminate the influence of the order in which the user interfaces are presented, and the potential resulting learning effects, we split the group in half; The first half started with the physical Domino interface, while the second half got to interact with the graphical version first.

Before interacting with the user interfaces, both the level of tactile appreciation and the level of holistic/analytic perception were measured. To judge the level of tactile appreciation, we used a 5 point Likert scale questionnaire consisting of statements related to tactile appreciation, i.e. "I enjoy assembling things" and "I possess well developed fine motor skills (dexterity)" (table 1).

The level of holistic or analytical perception was measured through the use of the framed-line test (figure 15). In this test, participants need to redraw a line either in absolute or in a relative sense. In the absolute task, participants need to mentally separate and ignore the size of the frame, a task better suited for analytical perception. The opposite holds for the relative task, where the frame and line need to be viewed as a whole, making this task better suited for a more holistic perception.

Having completed these tests, the participants were asked to re-enact a set of 5 different scenarios involving the lights in the IoT Sandbox living room (figure 14), namely "You just woke up", "Leaving the house", "Arriving home", "Having friends over" and "Going to bed". The descriptions associated with these scenarios ranged from simple: "You are leaving the house and want to turn all the lights off", to more complex: "You are having friends over and want to create a cosy environment".

The set of scenarios were repeated for both user interfaces, followed by a System Usability Scale (SUS) [3] combined with a set of questions related to the aesthetic appeal (table 2). Lastly, the tests concluded with several open questions to promote an open verbal evaluation of the perceived usability using questions including "If you would have to use one of these interfaces in your home, which one would it be (and why)?" and "Which interface did you enjoy using the most?".

Table 1. Tactile appreciation questionnaire

1. Friends would describe me as a technical person

2. I enjoy assembling things

3. When something brakes people often ask me to take a look.

4. I like to build things myself instead of buying them

5. Friends would describe my as being handy

6 . I enjoy building things using my hands

7. I posses well developed fine motor skills (dexterity)

Scaled: Stongle disagree [1] - [2] - [3] - [4] - [5] Strongly Agree

\section{Table 2. Interface evaluation questionnaire}

1. I found the interface aesthetically pleasing

2. I think that I would like to use this system frequently

3. If I owned this interface, I would give this interface a prominent location in my home

4. I found the system unnecessarily complex

5. I found the interface nice to look at

6. I thought the system was easy to use

7. I would imagine that most people would learn to use this system very quickly

8. I think that I would need the support of a technical

person to be able to use this system

9. I would describe this interface as beautiful

10. I found the various functions in this system were well integrated

11. I felt very confident using the system

12. I needed to learn a lot of things before I could get going with this system

13. I found the system very cumbersome to use

14. I thought there was too much inconsistency in this system

15. I consider this interface to be well designed
IoT Sandbox Living Room

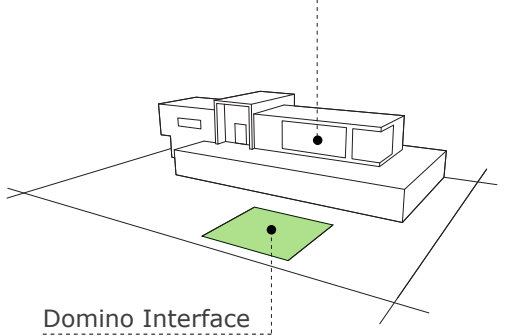

Figure 14. IoT Sandbox and Domino sketch

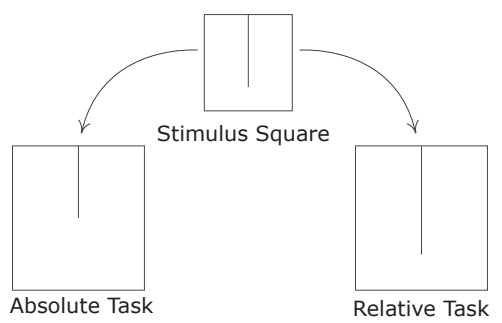

Figure 15. The Framed-Line test [16] is a graphical adaptation of the physica rod-and-frame test. The participant is first presented with a stimulus square, containing a line, and asked to remember the length of the line. The screen is then made blank for 5 seconds, after which a differently sized square ter which a diferenty sized square appears on a different location. The participant is asked to then redraw the line in the new square, either in absolute length (absolute task) or in relative length to the new square (relative task). 


\section{Results}

This study yielded scores from the tactile appreciation questionnaire, the SUS and the framed-line test as well as insights from the open questions. When we look at the SUS questionnaire results it is clear that the two interfaces score similarly on usability (graphical: $83 / 100$, physical $80 / 100$ ). This indicates that there was no inherent design bias present in the two user interfaces.

Although the tactile appreciation questionnaire scores did show a wide spread in tactile appreciation ranging from 11 to 96 out of 100 (averaging 53), the scores did not show a significant correlation between tactile appreciation and the SUS scores for either the physical or graphical Domino interface. The hypothesized positive correlation between higher tactile appreciation scores and a preference for the physical Domino interface is therefore not supported by our results.

A similar story holds for the correlation between the results from the framed-line test and the SUS scores for both interfaces, where there was again no significant correlation. Here it must also be noted that the framed line tests did not produce consistent results within participants.

Next to the quantitative measurements, there are also the qualitative results from the open questions. If we look at the answers to the open questionnaire we can see a clear split in preference for either type of interface. In total, 8 participants indicated that they preferred the physical interface, 6 participants preferred the graphical interface while 2 gave no preference.

Participants gave reasons for their preference, below we show two typical quotes, one from a preferred physical user interface, one from a preferred graphical user interface.

Participant A (prefers physical user interface):

"This one [physical Domino interface], because it gives you physical feedback. That is nicer for setting the intensity of the lights. [...] The feedback you can feel in your fingers. That works better than a slider."

Participant B (prefers graphical user interface):

"The iPad one [graphical Domino interface], it is easier to see the position of the lights on the screen."

\section{Discussion}

While we conducted an exploratory study, the limited number of participants as well as the relatively brief encounter that the participants had with the interfaces in the study limits the validity of our results. Notwithstanding, the results gave us two insights: First, our means of measuring tactile appreciation and the framed line test failed to demonstrate a relationship between the test scores and the SUS evaluations. The inconsistent outcomes of the framed line tests within single participants require further investigation, as it potentially indicates that our implementation of the tests might have been insufficient. Second, the responses to the open questions do show a clear split in preference for either the physical or the graphical user interface and yielded typical quotes to help us further in explaining this split. Below we discuss these two insights.

We expected that the preference for user interface would be explained by our measures. While we observed a clear split in preference, we could not demonstrate a relationship between the preference and the measures. To us this indicates that the measures are either not differentiating character traits (i.e., they do not effectively measure what we aimed to measure) or we had a (culturally and societally) homogeneous group of participants causing similar results. While we cannot be certain of either possibility, the fact that we recruited our 
participants from the student population of our university of technology (excluding students from the department of Industrial Design) arguably might have contributed to our measures not being able to explain the split in preference.

Regarding the preference for user interface itself and the discussion around diversification of interaction style that follows from that, $\mathrm{HCI}$ literature has consistently explored alternative interaction styles and still does. Early work on tangible interaction [14] and embodied interaction [6] explored 'natural' interaction styles that leveraged (embodied) familiarity with the world. Later work emphasized concepts like 'resonance' [12] and the aesthetics of coupling between the digital and the pysical [4] as drivers for UX design. More recently concepts like soma-based design [13] and slow computing [17] argued that the way technology is interfaced has enormous impact on our being, reaffirming the need for multimodality and embodiment in interaction as well as arguing for a first-person perspective as a driver in UX design. Additionally, Grønbæk et al. bring forward the (social) context of use and argue that diversity in interaction style is needed to account for variations in (social) context [10]. While the marketplace seems to be converging on touchscreen and voice interaction, $\mathrm{HCI}$ literature offers important arguments why a diversification of interaction style has merit. Our work should be seen in this light, finding preference for interaction style offers another argument for diversification and understanding how such preference is constituted offers handles for UX design.

We observed different preferences for the interfaces and, when not finding explanations between our measures, looked for the answers to the open questions that the participants provided. We observed that the participants were able to verbalize their reasons for their preference and we (cautiously) conclude that the quotes refer to very different (but not opposite) qualities of the two different prototypes. To us this indicates that the preference for user interface is rather multi-dimensional. Reflecting on this, in light of the work we discussed in the previous paragraph, we speculate that the context of use, the first-person perspective of the user (including underlying values), the social situation as well as the personality of a user contribute to the preference for user interface and we see that as important guidance for our future research.

\section{Reflection and Future research}

In this pictorial, we have presented the design of two functionally equivalent user interfaces. The design process of the two user interfaces showed us how a physical user interface can inform a graphical user interface and vice versa. We feel that the user interfaces and their respective design processes are an important contribution in themselves. The other contribution is a more tentative one; we present an exploratory user study where we compare the two Domino interfaces. Our original hypothesis that personality traits would be correlated to a preference for user interface style was not confirmed. Either because of the correlation not existing, or because our measurements were not able to adequately measure the effect. Rather than insisting on a quantitative approach forward we feel that a qualitative approach is warranted. We see (tentative) proof for this in the quotes of the participants and together with the perspectives from $\mathrm{HCI}$ literature this will inform a qualitative approach in our future research.

\section{Acknowledgements}

We acknowledge Lesley Lock and Simon Bavinck in designing an early version of a light interface (as part of their research internship with the second author) that served as inspiration for the physical interface, and "Amatec" for their help with selecting suitable belaville springs for the clutch mechanism. 


\section{References}

1. Victoria Bellotti, Maribeth Back, W. Keith Edwards, Rebecca E. Grinter, Austin Henderson and Cristina Lopes. 2002. Making sense of sensing systems: five questions for designers and researchers. Proceedings of the SIGCHI conference on Human factors in computing systems Changing our world changing ourselves CHI 02 1, 1: 415-422. https://doi. org/10.1145/503376.503450

2. Peter H. Bloch. 1995. Seeking the Ideal Form: Product Design and Consumer Response. Journal of Marketing 59, 3: 16. https://doi.org/10.2307/1252116

3. John Brooke. 1996. SUS-A quick and dirty usability scale. Usability Eval. Ind., vol. 189, no. 194, pp. 4-7.

4. Lukas van Campenhout, Kristof Vaes, Joep Frens and Caroline Hummels. 2020. The aesthetics of coupling: An impossible marriage. International Journal of Design, 14(2), 1.

5. Lukas Desmond Elias Van Campenhout, Joep Frens, Caroline Hummels, Achiel Standaert, and Herbert Peremans. 2016. Touching the dematerialized. Personal and Ubiquitous Computing 20, 1: 147-164. https://doi.org/10.1007/s00779-016-0907-y

6. Paul Dourish. 2001. Where the action is (p. 28). Cambridge: MIT press.

7. Joep Frens, Mathias Funk, Bastiaan van Hout, and Joep Le Blanc. 2018. Designing the IoT Sandbox. 2018: 341-354. https://doi. org/10.1145/3196709.3196815

8. Joep Frens. 2006. Designing for Rich Interaction: Integrating Form, Interaction, and Function. Doctoral Dissertation, Eindhoven University of Technology, Eindhoven, the Netherlands. (retrievable from www. richinteraction.com)
9. Saul Greenberg and Michael Boyle. 2002. Customizable physical interfaces for interacting with conventional applications. UIST (User Interface Software and Technology): Proceedings of the ACM Symposium 4, 2: 31-40. https://doi. org/10.1145/571990.571991

10. Jens Emil Grønbæk, Mille Skovhus Knudsen, Kenton O'Hara, Peter Gall Krogh, Jo Vermeulen and Marianne Graves Petersen. 2020. Proxemics beyond proximity: Designing for flexible social interaction through cross-device interaction. In Proceedings of the 2020 CHI Conference on Human Factors in Computing Systems (pp. 1-14).

11. Eve Hoggan, Stephen A Brewster, and Jody Johnston. 2008. Investigating the Effectiveness of Tactile Feedback for Mobile Touchscreens. CHI 2008 Proceedings - Tactile and Haptic User Interfaces 1573-1582.

12. Caroline Hummels and Aadjan van der Helm. 2004. ISH and the search for resonant tangible interaction. In Personal and Ubiquitous Computing. 1 September 2004;8(5):385-8

13. Kristina Höök, Baptiste Caramiaux, Cumhur Erkut, Jodi Forlizzi, Nassrin Hajinejad, Michael Haller, Caroline C.M. Hummels, Katherine Isbister, Martin Jonsson, George Khut, Lian Loke, Danielle Lottridge, Patrizia Marti, Edward Melcer, Florian F. Müller, Marianne G. Petersen, Thecla Schiphorst, Elena M. Segura, Anna Ståhl, Dag Svanæs, Jakob Tholander, and Helena Tobiasson. 2018. Embracing first-person perspectives in soma-based design. In Informatics (Vol. 5, No. 1, p. 8). Multidisciplinary Digital Publishing Institute.

14. Hiroshi Ishii and Brygg Ullmer. 1997. Tangible bits: towards seamless interfaces between people, bits and atoms. In Proceedings of the ACM SIGCHI Conference on Human factors in computing systems ( $\mathrm{pp}$. 234-241).
15. BSEN ISO and BRITISH STANDARD. 2010. Ergonomics of human-system interaction.

16. Shinobu Kitayama, Sean Duffy, Tadashi Kawamura, and Jeff T Larsen. 2003. Perceiving an Object and Its Context in Different Cultures : A Cultural Look at New Look. Psychological Science 14, 3: 201-206.

17. Rob Kitchin and Alistair Fraser. 2020. Slow Computing: Why we need balanced digital lives. Bristol University Press.

18. Wendy E Mackay. 2000. Is Paper Safer? The Role of Paper Flight Strips in Air Traffic Control. ACM Transactions on Computer-Human Interaction 6, 4 : 311-340.

19. Richard E. Nisbett and Yuri Miyamoto. 2005. The influence of culture: Holistic versus analytic perception. Trends in Cognitive Sciences 9, 10: 467-473. https://doi.org/10.1016/j.tics.2005.08.004

20. Donald A Norman. 2002. Emotion \& Design: Attractive things work better. Interaction, July. https://doi.org/10.1145/543434.543435

21. Stephan A. G. Wensveen, J P Djajadiningrat, and C J Overbeeke. 2004. Interaction frogger: a design framework to couple action and function through feedback and feedforward. Proceedings of the 5th conference on Designing interactive systems: processes, practices, methods, and techniques: 177-184. https://doi.org/10.1145/1013115.1013140

22. Lesley Xie, Alissa N. Antle, and Nima Motamedi. 2008. Are tangibles more fun? Comparing Children's Enjoyment and Engagement Using Physical, Graphical and Tangible User Interfaces. Proceedings of the 2nd international conference on Tangible and embedded interaction - TEI '08: 191-198. https://doi.org/10.1145/1347390.1347433 
23. Oren Zuckerman and Ayelet Gal-Oz. 2013. To TUI or not to TUI: Evaluating performance and preference in tangible vs. graphical user interfaces. Interna-

tional Journal of Human Computer Studies 71, 7-8: 803-820.

https://doi.org/10.1016/j.ijhcs.2013.04.003

24. Google Nest Thermostat. Retrieved January 20, 2020 from https://store.google.com/nl/product/ nest_learning_thermostat_3rd_gen

25. Philips Hue Lights. Retrieved January 15, 2020 from https://www.meethue.com/

26. Samsung WW9000 Ecobuble Washing Machine.

Retrieved January 15, 2020 from https://www.samsung.com/uk/laundry/washer-ww10h9600ex/ 